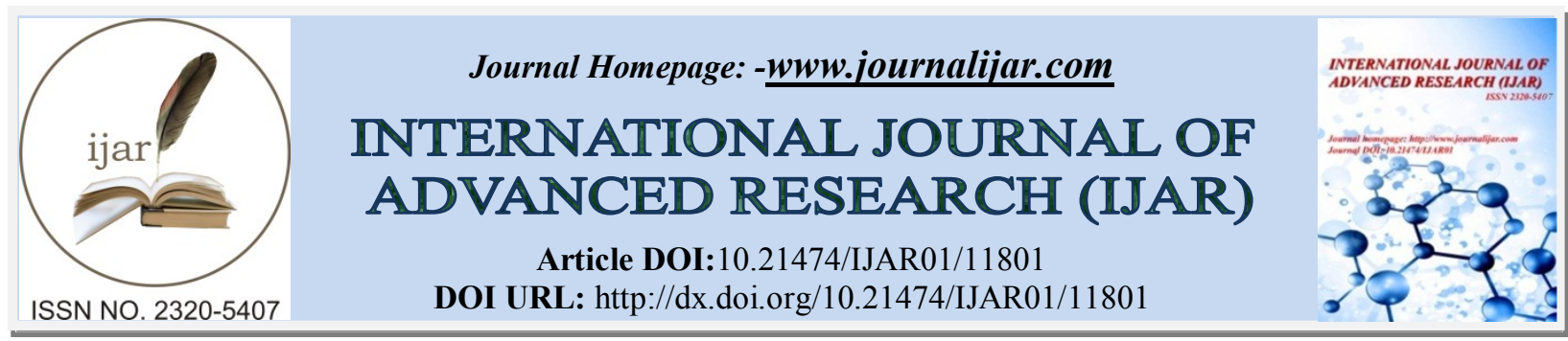

RESEARCH ARTICLE

DIAGNOSTIC ET TRAITEMENT DE L'ENDOMETRIOSE CHEZ L'ADOLESCENTE A PROPOS DE 160 CAS

\title{
Omar Laghzaoui
}

Université Sidi Mohammed Ben Abdellah Faculté de, Médecine et de Pharmacie. Fès Maroc.

\section{Manuscript Info}

(.........................

Manuscript History

Received: 26 July 2020

Final Accepted: 31 August 2020

Published: September 2020

Key words:-

Teenager, Pelvic Pain, Endometriosis

\begin{abstract}
Endometriosis is a chronic gynecological pathology related to the presence of hormonally-dependent endometrial tissue outside the uterus. Its frequency in the adolescent is not negligible with a symptomatology dominated by pelvic pain twisted on the account of dysmenorrhea. Pelvic ultrasound is a basic examination for diagnostic guidance; magnetic resonance imaging is more sensitive to visualizing deep endometriosis. Surgical and medical treatments are complementary. The evolution is marked by frequent recurrences in the teenager which can hinder the social life as well as the obstetric prognosis.
\end{abstract}

Copy Right, IJAR, 2020,. All rights reserved.

\section{Introduction:-}

L'endométriose est une pathologie gynécologique multifactorielle caractérisée par la présence de tissu de l'endomètre hors de la cavité utérine. C'est une maladie hormono-dépendante de la femme en période d'activité génitale et dont l'apparition peut se voir à l'âge de l'adolescence avec un retentissement physique et psychique important.

Notre étude rétrospective porte sur un effectif de 160 adolescentes atteinte d'endométriose prises en charge à l'hôpital militaire Moulay Ismail de Meknès sur une période de 10 ans.

Le but de ce travail épidémiologique est de lever le voile sur les caractéristiques sémiologiques de cette pathologie à l'âge de l'adolescence précisant les circonstances de découverte et les moyens para-cliniques utilisés pour asseoir le diagnostic ainsi que la fréquence du siège de la lésion et en fin les procédés thérapeutiques optimaux pour cette tranche d'âge.

\section{Matériels:-}

Cette étude rétrospective a été réalisée sur une période de dix ans, de janvier 2008 à décembre 2018, au service de gynécologie-obstétrique de l'hôpital militaire Moulay Ismail Meknès.

Les critères d'inclusion sont cliniques, radiologique et histologiques des patientes en âge de l'adolescenceentre 14 et 19 ans porteuses d'endométriose. L'étude des dossiers a pu collecter 160 adolescentes parmi 1140 patientes prises en charge pour l'endométriose.

Corresponding Author:- Omar Laghzaoui

Address:- Université Sidi Mohammed Ben Abdellah Faculté de, Médecine et de Pharmacie. Fès Maroc. 


\section{Résultats:-}

Les adolescentes représentent $14 \%$ de la pathologie endométriosique diagnostiquée en dix ans avec un âge moyen de 16 ans et des extrêmes de 14 ans à 19 ans. 120 patientes sont célibataires 40 sont mariées dont 20 n'ayant pas eu de gestation au-delà de deux ans de vie de couple soit 50\% d'infertilité. L'âge moyen des ménarches de nos patientes est de 10 ans avec des extrêmes de 08 et 12 ans. 15 adolescentes sont primipares, trois deuxième pare et deux enceinte au deuxième trimestre.

\section{Etude clinique :}

Le motif principal de consultation est la douleur pelvienne chez 120 patientes ce qui représente $75 \%$ ensuite c'est l'irrégularité du cycle menstruel, l'infertilité et l'aménorrhée primaire.La douleur siège surtout au niveau de la fosse iliaque droite chez 55 patientes, la fosse iliaque 50 patientes, hypogastrique 43 patientes, 4 en péri ombilicale, deux patientes accusent une dyspareunie et 6 adolescentes n'arrivent pas à préciser le siège de la douleur.

L'intensité de la douleur quantifiée sur une échelle de 1 à 10 trouve une douleur légère (de 1 à 4 ) chez 30 patientes, une douleur modérée (de 5 à 6 ) chez 120 adolescentes, et douleur sévère invalidantes (de 7 à 10) chez 10 patientes cause d'absentéisme scolaire fréquent. La dysménorrhée est présente chez 134 patientes dont 60 présentent un syndrome prémenstruel. Les signes accompagnateurs les plus souvent retrouvés sont les arthralgies, les lombalgies, les céphalées et les vomissements (Tableau 1).

Le cycle menstruel est régulier chez 20 patientes, 134 ont un cycle irrégulier et 6 patientes avaient une aménorrhée primaire au-delà de 19 ans.

L'examen clinique découvre des lésions ombilicales pathognomoniques d'endométriose pariétale chez 4 patientes sous forme de nodule bleu noirâtre augmentant de volume pendant les menstruation avec suintement séro-sanglant (Figure 1).

L'examen clinique objective à la palpation abdomino-pelvienne des masses mal limitées, irrégulières rémittentes, légèrement sensibles à douloureuses chez 102 adolescentes. La perception d'un empâtement hypogastrique sans masse palpable chez 2 patientes. L'examen vulvo-vaginal réalisé chez 40 adolescentes mariées met en évidence une imperforation de l'hymen chez deux patientes, un nodule douloureux de la face postérieure du vagin chez une adolescente et un kyste clitoridien (Figure 2) plus une lésion bleuâtre au cul se sac antérieur du vagin chez une patiente (Tableau 2).

\section{Bilan para Clinique:}

L'échographie pelvienne objective des images kystiques unique ou multiples polylobés chez 111 patientes: la localisation ovarienne intéresse 85 patientes (Figure 3) alors que 24 adolescentes présentent des images d'échogènessité hétérogène comblant le cul de sac de Douglas en dehors des ovaires, deux patientes présentent des images kystiques hétérogènes refoulant la vessie en avant et un utérus rétro-versé fixé chez 15 adolescentes, un hématocolpos chez deux patientes et L'échographie pelvienne est normale chez 59 adolescentes.

La TDM pelvienne réalisée chez 140 patientes confirme le siège ovarien des lésions chez 95 adolescentes, la localisation pelvienne en dehors des ovaires chez 28 patientes dont deux ont un hématocolpos avec image de rétention utéro-vaginale, le siège inter vésico-utérin chez 6 malades tandis que 21 patientes présentent une TDM normale malgré les douleurs cycliques (Figure 4).

La réalisation de l'IRM chez 21 adolescentes permet de mètre en évidence 9 patientes présentent une lésion d'endométriose au niveau du ligament large, 6 au niveau des utéro-sacrés 4 localisations au niveau du ligament lombo-ovarien confluant avec des kystes ovariens et 2 localisations au niveau de la cloison recto-vaginale (Figure $5)$.

\section{Traitement chirurgicale:}

L'ensemble des patientes ont bénéficiées d'un traitement chirurgical. La cœlioscopie à visé diagnostic et thérapeutique est réalisé chez 156 malades avec 15 conversion en laparotomie pour lésions étendues et saignant à la dissection. 
La résection des kystes ovariens et la destruction des lésions endometriosiques pélvi-peritonéales est réalisée chez 154 patientes, 4 patientes ont bénéficiées d'exérèse ombilicale et deux patientes une destruction du nodule vaginale après biopsie. L'hymeniotomie est réalisée chez deux patientes qui ont un une imperforation d'hymen avec hématocolpos dont une présente un kyste vulvaire. L'étude anatomopathologique réalisée chez toutes les patientes confirme le diagnostic d'endométriose.

\section{Aspects macroscopique et localisation des lesions:}

Lésions kystiques sans plan de clivage donnant issu à un liquide marron épais à localisation ovarienne chez 80 patientes, le même aspect à localisation pelvienne chez 31 adolescentes.

1. Les lésions rouges sous forme de bulles au niveau de la base de l'ombilic chez 4 patientes.

2. Les plaques marron avec épaississements tissulaire blanchâtres à localisation épiploique et pariétale chez 35 patientes.

3. Le péritoine cribriforme et hypervascularisé chez 26 patientes.

4. des brèches péritonéales chez 5 patientes.

5. des adhérences multiples chez 121 patientes.

\section{Classification des lesions:}

L'évaluation per-opératoire de la sévérité de l'endométriose objective selon les critères révisés de la classification de l'American Society of Reproductive Médicine (ASRM) [49] : 50 patientes stade I ; 80 adolescentes stade II; 24 patientes stade III et 6 patientes stade IV.

\section{Traitement medical:}

71 adolescentes ont bénéficiées d'un traitement médical complémentaire avec une surveillance clinique et radiologique allant de 3 à 6 mois. Le traitement médical complémentaire est instauré chez les patientes ayant des lésions importantes et chez qui le traitement chirurgical est insuffisant (Tableau 3).

\section{L'évolution:}

L'évolution est marquée par la disparition de la douleur ainsi que des lésions chez 102 adolescentes. Persistance d'une dysménorrhée chez 45 sans lésion apparente à l'IRM. Récidive après six mois malgré le traitement chirurgicale et médical chez 41 patientes, la reprise chirurgicale suivie de traitement médical au decapeptyl permettant une évolution favorable chez 11 patientes. 30 patientes sont en cours d'évaluation d'évolution.

L'évolution obstétricale est marquée par l'obtention de 12 grossesses sur 20 patientes suivies pour stérilité après traitement de l'endométriose et induction au citrate de clomifène.

L'issu des grossesses a donné naissance à dix nouveaux nés bien portant, neuf gestantes ont accouchées par voie basse et une patiente a accouchée par césarienne pour souffrance fotale. Deux patientes sont enceintes au deuxième trimestre.

\section{Discussion:-}

\section{Caractéristiques démographiques:}

L'endométriose n'épargne pas l'adolescente avec un pic d'âge de 17 ans et une augmentation de l'incidence avec l'âge. Dans notre étude, l'âge des patientes est variable allant de 14 ans à 19 ans avec un pic de $65 \%$ qui se situe à 17 ans $[3,7]$.

\section{Motif de consultation:}

Chez les adolescentes les circonstances de découverte de l'endométriose sont multiples prédominées par la douleur pelvienne chronique qui est le motif principal de consultation avec une fréquence variable selon les études entre 25 et $70 \%$, dans notre étude la fréquence est de $75 \%$.

La dysménorrhée vient au second plan et surtout le caractère sévère de la douleur qui est un signe d'alarme. Selon la littérature une patiente sur deux ayant une dysménorrhée sévère, invalidante avec retentissement sur l'activité usuelle est porteuse d'une endométriose.

L'infertilité représente un motif peu fréquent à cette tranche d'âge ou les patientes sont encore célibataires [4,9,14]. 


\section{Les facteurs prédisposant à l'endométriose:}

Les ménarches précoces (avant 11 ans) paraissent être un facteur de risque important d'endométriose ainsi qu'un cycle menstruel inférieur à 28 jours et des menstruations de plus de 7 jours.

Le risque de développer une endométriose diminue avec le nombre de grossesse et l'âge précoce de gestation ainsi la grossesse joue un rôle prophylactique vis-à-vis de l'endométriose.

Il existe une prédisposition génétique à l'endométriose qui est plus élevé lorsqu'il existe des antécédents familiaux ( 6 fois en cas d'antécédents chez la mère).

Lamb pense surtout aux habitudes familiales de vie qui expliquent cette différence plutôt que le facteur génétique en somme La notion d'une histoire familiale est donc un argument en faveur du diagnostic $[2,3,10]$.

\section{L'examen Clinique:}

L'examen clinique chez l'adolescente peut être difficile mais essentiel nécessitant un abord délicat accompagné d'explications de la part de l'examinateur, il est utile de le réaliser pendant les règles ce qui permet d'augmenter sa sensibilité.

Le toucher vaginal uni digital chez les patientes vierge couplé au toucher rectal permet d'apprécier le volume de l'utérus sa mobilité et préciser les caractéristiques cliniques d'une masse latéro-utérine, le réveil d'une douleur des ligaments utéro-sacrés ainsi que l'existence d'un nodule de la cloison recto-vaginale. L'examen au spéculum pour vierge est bien sûr très important permettant de découvrir d'endométriose cervicale ou vaginale $[5,7]$.

Dans notre série l'examen clinique a permis la découverte d'endométriose pariétale chez 4 patientes, deux localisations vaginales et deux imperforation de l'hymen dont une présente un kyste vulvaire.

\section{Les examens para-cliniques:}

Echographie pelvienne:

L'échographie pelvienne sus ou endovaginale est le premier examen radiologique a réalisé vu sa sensibilité en matière du kyste endometriosique ovarien qui est de $83 \%$ et une spécificité de $98 \%$. L'image kystique se présente sous une forme arrondis à contours réguliers avec une paroi fine et un contenu généralement homogène. L'échographie pelvienne dois rechercher également d'autre localisation en dehors des ovaires, un utérus rétro-versé fixé ou un hematocolpos. Dans notre série 111 adolescentes ont des images polylobés à l'échographie suspectant une endométriose non ovariennes, également cet exploration pelvienne a objectivé un utérus rétro-versé chez quinze patientes et un hématocolpos auprès de deux patientes.

La normalité de l'échographie n'exclue pas le diagnostic d'endométriose, d'autant que les lésions péritonéales et d'autres localisations profondes sont en général ignorées par cet examen, ce qui est le cas pour 59 de nos patientes qui ont une échographie totalement normale. $[1,4,6]$.

\section{TDM et IRM pelviennes}

La tomodensitométrie est un examen peu performant en matière d'endométriose aussi bien que la visualisation des structures du petit bassin. L'imagerie par résonance magnétique est l'examen de choix qui permet de visualiser la lésion endométriosique notamment au niveau des zones peu accessibles à l'échographie (les ligaments utéro-sacrés et la cloison-recto-vaginale). C'est un examen qui permet d'établir la cartographie des lésions et le suivie évolutif.

Stratton et al retrouvent une sensibilité de $69 \%$ et une spécificité de $75 \%$ pour une corrélation imagerie/histologie. Bazot rapporte une sensibilité et spécificité de 90 et $91 \%$ en termes de corrélation imagerie/cœlioscopie [1].

\section{Colioscopie :}

La cœlioscopie est l'examen de référence pour le diagnostic ainsi que le traitement de l'endométriose, elle permet de voir les lésions endométriosiques et de réaliser une biopsie qui seule affirme le diagnostic. Cependant, la cœlioscopie ignore les lésions d'endométriose sous péritonéales profondes. La réalisation d'une cœlioscopie est recommandée par le Collège américain d'obstétrique et de gynécologie (ACOG) pour toutes les adolescentes de moins de 18 ans présentant des algies pelviennes résistantes à un traitement médical $[5,13,15]$. 


\section{La Stadification :}

La majorité des adolescentes qui présentent l'endométriose sont soit au stade I (61 à $92 \%)$, soit au stade II de la maladie ( 8 à $30 \%$ ) selon les critères révisés de la classification de l'American Society of Reproductive Médicine (ASRM) $[8,17]$. Notre étude objective l'inverse $50 \%$ des patientes en stade II et $31 \%$ en stade I.

\section{La Localisation :}

Selon Brosens. Les adolescentes présentent plus fréquemment des lésions atypiques au niveau du péritoine et de la surface ovarienne et rarement des lésions de la cloison recto-vaginale. Les lésions sont caractérisées par une angiogenèse importante une hypervascularisation [8]. Ce n'est pas le cas de nos patientes qui présentent des lésions intra-ovariennes typiques et deux patientes ont une localisation vaginale.

\section{Traitement :}

Le choix de la thérapeutique instaurée est en fonction du degré d'atteinte, et du désir de grossesse en respectant la balance du bénéfices/risque, Les modalités thérapeutiques sont médicaux, chirurgicaux ou les deux combinées.

\section{Traitement médical exclusif :}

Les progestatifs ont réellement été les premiers traitements utilisés grâce à leurs actions anti-œstrogénique et antigonadotrope, et de leur forte affinité pour les récepteurs à la progestérone. L'usage des anti-inflammatoires non stéroïdiens est établi sur les effets antiprostaglandines sachant que les lésions endométriosiques produisent d'une manière active de la prostaglandine qui est à la base de la douleur.

L'association des progestatifs avec des AINS et des antalgique été le traitement de première ligne envisager par Laufer chez les adolescentes présentant des dysménorrhées avec un succès de $60 \%$ [13].

Le danazol ou les agonistes de la gonadotrophin releasing hormone $(\mathrm{GnRH})$ posent le problème majeur de l'androgénicité, des troubles métaboliques et les risques de déminéralisation osseuse ceci limite leurs utilisation chez l'adolescente $[6,13,15]$.

Selon la HAS décembre 2017 l'usage des oestro-progestatifs chez les adolescentes après preuve formelle d'endométriose est de mise mais pour des lésions minimes.

\section{Traitement chirurgicale:}

Il Consiste en la résection ou la destruction la plus complète possible des lésions endométriosiques pour un meilleur résultat. La voie d'abord coelioscopique ou laparotomique est dicté par le site de la localisation l'étendue et le nombre des lésions. La voie cœlioscopique quand elle est possible est à préférer du fait de la diminution du coût, des douleurs post-opératoire, la durée d'hospitalisation et de récupération plus rapide. Quant aux lésions profondes étendues et multiples une chirurgie conventionnelle offre une meilleure vision pour détruire les lésions. [13]

L'ensemble de nos patientes ont bénéficié d'un traitement chirurgical. La voie coelioscopique à été réalisé chez 156 adolescentes avec 15 conversions en laparotomie devant les difficultés dues aux adhérences et au saignement.

\section{Traitement médical post chirurgie :}

Le choix du traitement médical complémentaire doit être guidé par les résultats de la chirurgie les contre-indications, les effets indésirables potentiels, le désir de conception de la patiente. Les traitements hormonaux complémentaires sont :

La contraception orale oestro-progestatif, les micro-progestative orale au désogestrel ou le diénogest, l'implant à l'étonogestrel et le decapeptyl $[13,15]$.

\section{L'évolution :}

L'ensemble des publications accorde une corrélation entre le stade des lésions et le résultat obtenue après traitement chirurgicale avec d'exérèse complète des lésions. Stavroulis décrie l'obtention de bons résultats du traitement chirurgical à court et moyen terme chez des adolescentes souffrant de douleurs pelviennes chroniques résistantes au traitement médical. Concernant les réinterventions chirurgicales pour récidives exposent à davantage de difficultés du fait des adhérences avec des résultats positifs ne dépassant pas 16\%.Dans notre étude la récidive après chirurgie et traitement médical concerne $8 \%$ de nos patientes [5,7]. 


\section{Conclusion:-}

La fréquence de l'endométriose chez l'adolescente parait sous estimé alors que son incidence est proche de celle de la femme plus âgée. La dysménorrhée et la dyspareunie sont des symptômes d'éventuelle endométriose qu'il faudrait diagnostiquer. Les examens radiologiques orientent le diagnostic l'étude anatomopathologique confirme la pathologie. Le traitement chirurgical l'emporte sur le traitement médical qui lui peut être complémentaire pour améliorer l'évolution. L'objectif du traitement est l'arrêt de la progression de la maladie, d'éradiquer la douleur, et préservé la fertilité.

\section{Bibliographie:-}

1. G, Bazot M Moura APC, Ribeiro HSAA, Bernardo WM, Simões R, D'Ippolito, Ribeiro PAAG Correction: Accuracy of transvaginal sonography versus magnetic resonance imaging in the diagnosis of rectosigmoid endometriosis: Systematic review and meta-analysis. PLoS One. 2019 Aug 16;14(8).

2. Harel, Z. «A contemporary approach to dysmenorrhea in adolescents ». Pediatr Drugs,vol. 2002; 4: 797-805

3. Audebert A. Caractéristiques de l'endométriose de l'adolescente : à propos d'une série de 40 cas. Gynécol Obstet Fertil $2000 ; 28: 450-4$.

4. Johnston JL, Reid H, Hunter D. Diagnosing endometriosis in primary care: clinical update. Br J Gen Pract. 2015; 65(631):101-2.

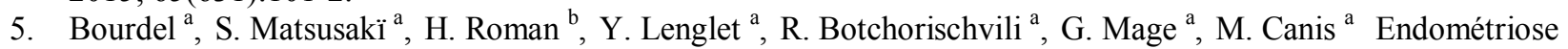
chez l' adolescente 2006

6. Dessole M, Melis GB, Angioni S. Endometriosis in adolescence. Obstet Gynecol Int 2012; 2012 : 869191 .

7. Bai SW, Cho HJ, Kim JY, Jeong KA, Kim SK, Cho DJ, et al. Endometriosis in an adolescent population: the Severance hospital in Korean experience. Yonsei Med J 2002; 43:48.

8. Adamson GD, Pasta DJ. Endometriosis fertility index: the new, validated endometriosis staging system. Fertil Steril. 2010;94:1609-1615.

9. Parazzini F, Esposito G, Tozzi L, Noli S, Bianchi S. Epidemiology of endometriosis and its comorbidities. Eur J Obstet Gynecol Reprod Biol. 2017; 209:3-7.

10. Hemmings R, Rivard M, Olive DL, Poliquin-Fleury J, Gagné D, Hugo P, et al. Evaluation of risk factors associated with endometriosis. Fertil Steril. 2004; 81(6):1513-21.

11. Sanctis V, Matalliotakis M, Soliman AT, Elsefdy H, Di Maio S, Fiscina B. A focus on the distinctions and current evidence of endometriosis in adolescents. Best Pract Res Clin Obstet Gynaecol. 2018; 1-13.

12. Closon F, Brichant G, Tebache L, Pinzauti S, Nisolle M. L'endométriose de l'adolescente. Médecine Reprod. 2013; 15(3):228-33.

13. Quibel A, Puscasiu L, Marpeau L, Roman H. Les médecins traitants devant le défi du dépistage et de la prise en charge de l'endométriose : résultats d'une enquête. Gynécologie Obstétrique Fertil. 2013;41(6):372-80.

14. Gover, S. « Pelvic pain in the female adolescent ». Aust Fam Physician. 2006; 35: 850-853

15. PONCELET C., DUCARME G. « Prise en charge de l'endométriose : les bonnes pratiques de la coelioscopie diagnostique ». J. Gynécologie Obstétrique Biol. Reprod. [En ligne]. avril 2007. Vol. 36, n², p. 135-140

16. Haute Autorité de santé GL. Recommandation de bonne pratique - Prise en charge de l'endométriose Recommandations pour la pratique clinique. 2017;1-39.

17. Johnson NP, Hummelshoj L, Adamson GD, Keckstein J, Taylor HS, Abrao MS, et al. World Endometriosis Society consensus on the classification of endometriosis. Hum Reprod. 2017; 32(2):315-24. 